\title{
Buchbesprechung
}

\section{Birgit Spinath (Hrsg.). (2014). Empirische Bildungsforschung: Aktuelle Themen der Bildungspraxis und Bildungsforschung. Ber- lin: Springer. 158 Seiten, ISBN-10: 364241 6977 (€ 24,99).}

Die Verfügbarkeit von Lehrwerken über psychologische Themenfelder ist heute möglicherweise besser als je zuvor. Viele Verlage haben eigene Reihen an Lehrwerken aufgelegt, die zum größten Teil inhaltlich hervorragend gelungen sind. Angesichts dieser Fülle kommt es selten vor, etwas genuin Neues in die Hände zu bekommen. Ein solches innovatives Konzept legt Spinath (2014) mit ihrem Werk über empirische Bildungsforschung vor. Sie bedient sich anders als die anderen Lehrwerke eines journalistischen Zugangs: Aktuelle und in der öffentlichen Diskussion präsente Themen werden als provokante Thesen formuliert, die den Ausgangspunkt für Experteninterviews bilden. Die Interviews selbst wurden von Studierenden geplant, durchgeführt, per Video aufgezeichnet und transkribiert. Die Videos sind zum großen Teil auf einem Videoportal bei Springer verfügbar. Im Buch werden die Interviews durch weiterführende Informationen ergänzt. Das Buch gliedert sich in drei große Themenkomplexe:

\section{Bildungsqualität von Schule}

Ein starker Fokus des ersten Themenkomplexes liegt in der Fragestellung nach Ausmaß und Ursachen von Leistungsunterschieden zwischen Jungen und Mädchen in der Schule (Interview mit Ursula Kessels) und welche Rolle dabei die weibliche Dominanz im Bildungssystem mutmaßlich spielt (Interview mit Martin Neugebauer). Petra Stanat und Anand Pant reflektieren über die Frage, welche Auswirkungen PISA auf die Organisation unseres Bildungswesens und die Unterrichtskultur hatte. Mareike Kunter wiederum richtet unter Bezugnahme aufCOACTIV den Blick auf konkrete Unterrichtsprozesse.

\section{Soziale Ungleichheit}

Der zweite große Themenblock fragt nach individuellen Unterschieden in Leistung und Bildungsbeteiligung und deren Ursachen. Inwieweit soziale Ungleichheit in Ungerechtigkeit mündet und welche
Rolle bei der Entstehung von Ungleichheit genetische Unterschiede spielen, wird von Martin Diewald und Rainer Riemann behandelt. Elsbeth Stern richtet ihren Fokus dagegen auf den Einfluss der Umwelt, auf die Intelligenzentwicklung und die Rolle einer reichhaltigen Lernumwelt. Sie diskutiert prototypische Fehlkonzepte zur Erblichkeit von Intelligenz. Soziale Disparitäten und die Frage, in wieweit das Schulsystem diese Unterschiede weiter verstärkt, ist Gegenstand des Interviews mit Ulrich Trautwein. Der letzte Beitrag des Themenblocks reflektiert die Kosten verpasster Bildungschancen. Als einen der drängendsten Bereiche des deutschen Bildungssystems identifiziert Katharina Spieß die frühkindliche Bildung.

\section{Qualität hochschulischer Bildung inklusive Lehrerbildung}

Die letzten beiden Beiträge des Lehrwerks betrachten die Auswirkungen der Bologna-Reform und die Eignung von Kandidaten für Lehramtsstudiengänge (Ulrich Teichler \& Ewald Terhart).

Das Buch hat einige klare Einschränkungen: Je nach Fähigkeit des Interviewers oder der Interviewerin variiert die Qualität der Fragestellung. Auch reicht die Qualität der Videoaufzeichnungen sicher nicht an professionelle Reportagen heran und sie variiert zudem zwischen verschiedenen Beiträgen deutlich. Dennoch kommt gerade der Interviewstil der Lesbarkeit und Verständlichkeit der Inhalte sehr zugute: Die Expertinnen und Experten konnten im Gespräch aus ihrem Wissensfundus schöpfen und Missverständnisse und Fehlkonzepte unmittelbar ausräumen. Dabei bleibt die Darstellung kompakt und ermöglicht die schnelle Gewinnung eines Überblicks. Für weitere Auflagen wäre dem Lehrwerk zu wünschen, dass noch weitere, spannende Themen aufgegriffen werden, die die inhaltliche Basis weiter verbreitern.

Insgesamt liegt diesem Lehrwerk ein sehr innovatives Konzept zugrunde und es stellt eine hervorragende Ergänzung wissenschaftlicher Publikationen in der Lehramts- und Psychologieausbildung dar.

\author{
Wolfgang Lenhard \\ Uni Würzburg \\ DOI 10.2378/peu2014.arto6d
}

